\title{
POLYMERS IN COMPULSORY SECONDARY EDUCATION AND HIGH SCHOOL IN SPAIN: ANALYSIS OF TEXTBOOKS AND TEACHERS' OPINION
}

\author{
SERRANO-AROCA, Ánge $1^{1^{*}}$; SOLAZ-PORTOLÉS, Joan Josep ${ }^{2}$ \\ "Universidad Católica de Valencia "San Vicente Mártir" (UCV), Facultad de Veterinaria y Ciencias \\ Experimentales, Departamento de Ciencias Aplicadas y Tecnológicas, C/ Guillem de Castro 94, 46003 Valencia \\ (Spain) \\ ${ }^{2}$ Universitat de València, Facultat de Magisteri, Departament de Didàctica de les Ciències Experimentals i \\ Socials, Avinguda dels Tarongers 4, 46022 Valencia (Spain) \\ ${ }^{*}$ Autor correspondente \\ e-mail: angel.serrano@ucv.es
}

Received 19 April 2015; received in revised form 25 July 2015; accepted 05 August 2015

\section{RESUMEN}

A pesar de que el estudio de los polímeros está presente en el currículo de la educación secundaria española, todos los indicios parecen indicar que este tema no se aborda adecuadamente en el aula. Por esta razón, este trabajo se centra en dos factores clave en la enseñanza y aprendizaje de los polímeros en la educación secundaria: el profesorado y los libros de texto. Se ha utilizado una metodología cualitativa, basada en entrevistas semiestructuradas y un análisis del contenido de libros de texto. Han participado seis profesores de educación secundaria, cuya opinión sobre la introducción de polímeros en el aula ha sido recogida mediante entrevistas semiestructuradas. El análisis de los contenidos básicos sobre polímeros de varios libros de texto muy utilizados en España ha sido llevado a cabo por medio de un cuestionario de valoración de los contenidos. Los resultados obtenidos en relación con los polímeros sugieren que: a) El profesorado les dedica poco tiempo en el currículo; b) Los libros de texto no tratan una buena parte de los contenidos básicos; y c) El bajo nivel de conocimientos de los estudiantes se justifica por a) y b).

Palabras clave: Investigación cualitativa, sustancias macromoleculares, textos educativos, escuela secundaria, docentes.

\begin{abstract}
Even though the study of polymers is included in the school secondary curriculum in Spain, all the signs are that this topic is not being adequately addressed in the classroom. For this reason, this paper focuses on two key factors for polymers teaching and learning at secondary level: teachers and textbooks. A qualitative methodology, based on semi-structured interviews and a content analysis of textbooks, has been used. Six High School teachers have participated in this research. Attempts have been made to find out teachers' opinion about introducing contents of polymers in the classroom by means of semi-structured interviews. The content analysis of textbooks used in many schools of Spain has been carried out through the application of a assessment questionnaire. The results obtained as regards the polymers suggest that: a) Teachers provide only a limited amount of time for this topic in the curriculum; b) Textbooks do not include a large part of basic contents; and c) The low level of knowledge of students is justified by a) and b).
\end{abstract}

Keywords: Qualitative research, macromolecular substances, schoolbooks, secondary school, teaching staff. 


\section{INTRODUCCIÓN}

El estudio de los materiales se introduce en España por primera vez en el currículo del área de las ciencias de la naturaleza de la Educación Secundaria Obligatoria (de ahora en adelante ESO, que va desde los 12 hasta los 16 años) en 1992. La experiencia desde entonces es que ha sido difícil consolidar este contenido básico en el currículo de ciencias de naturaleza en la ESO. En este sentido, se ha constatado que el estudio de los materiales tiene una escasa presencia en el currículo actual (De Pro, 2007), debido a las dificultades del tiempo disponible (Caamaño, 2009). En la educación secundaria posobligatoria (Bachillerato) los materiales van ganando protagonismo, aunque algunos de ellos tengan poca presencia. Así, por ejemplo, los conceptos y procedimientos relacionados con los materiales poliméricos son exiguos en el currículo de Química de Bachillerato (Camaño, 2006).

En el currículo oficial vigente en España pueden encontrarse contenidos sobre polímeros en dos asignaturas de la ESO, Tecnología (obligatoria a los 15 años) y Física y Química (optativa a los 16 años); y en tres de Bachillerato, Ciencias para el Mundo contemporáneo (obligatoria a los 17 años), Tecnología Industrial (optativa a los 17 años), y Química (optativa a los 18 años).

En una reciente publicación (SerranoAroca \& Solaz-Portolés, 2014) se ha puesto en evidencia que el nivel de conocimientos sobre materiales poliméricos adquirido por los estudiantes al acabar la ESO (16 años) está muy lejos de ser el óptimo, y no mejora al finalizar el Bachillerato científico (18 años).

Nuestro interés en el presente estudio se centra en investigar las razones que justifiquen el bajo nivel de conocimientos sobre estos materiales de los estudiantes de educación secundaria (ESO y Bachillerato). Para ello nos fijaremos en dos pilares básicos de los procesos de enseñanza/aprendizaje: el profesor y el libro de texto.

En relación con el papel fundamental del profesor, cabe destacar, a título de ejemplo, que el éxito educativo finlandés está basado en gran parte en la figura del profesor como elemento clave (Enkvist, 2010). En cuanto a los libros de texto, es bien conocida su extraordinaria influencia en la enseñanza de las ciencias (Yore, 1991). De hecho, son uno de los recursos didácticos de apoyo más utilizados por los profesores em la educación secundaria, y determinan la naturaleza de la actividad científica en el aula (Duarte, 1999). Esta circunstancia se ha subrayado también en otros trabajos (Campanario, 2001; Perales \& Jiménez, 2002), en donde se señala que los libros de texto plasman el currículo que el docente intentará desarrollar. En definitiva, los libros de texto son una ayuda que puede facilitar el proceso de enseñanza y aprendizaje, aunque no aporten, en sí mismos, garantías para hacerlo (De Pro et al., 2008).

Así pues, nuestro problema a investigar es: ¿se puede justificar el bajo nivel de conocimientos de los estudiantes de secundaria sobre polimeros en el proceder del profesorado y el tratamiento que ofrecen los libros de texto?

\section{METODOLOGÍA}

El diseño de la investigación es cualitativo y se utilizan la entrevista clínica y el análisis de libros de texto como técnicas de recogida de la información. La entrevista clínica se ha revelado como un instrumento de gran valor en la investigación educativa, particularmente en la investigación en la didáctica de las ciencias (Moreira \& Lang da Silveira, 1993). En este trabajo se han llevado a cabo entrevistas semiestructuradas (Mayan, 2001). Por su parte, el análisis de libros de texto se ha convertido en un instrumento eficiente para la investigación de los procesos de enseñanza/aprendizaje en ciencias (González \& Sierra, 2004).

\subsection{Sujetos participantes}

Han participado en las entrevistas seis profesores de un centro de educación secundaria público de la ciudad de València (España). Cuatro pertenenecen al Departamento de Física y Química, y tienen más de 20 años de experiencia docente. Los otros dos profesores son del Departamento de Tecnología, y tienen más de 12 años de experiencia docente.

\subsection{Materiales}

Los materiales utilizados en este trabajo son: los libros de texto analizados (Anexo), el guión de preguntas utilizado en la entrevista de profesores (Tabla 1), y el cuestionario (o planilla) 
de análisis de los libros de texto (Tabla 2).

Como puede verse en la Tabla 1 las preguntas realizadas en las entrevistas a los profesores están relacionadas con seis cuestiones básicas sobre la enseñanza de los polímeros: dedicación en el currículo, necesidades para su enseñanza, formación del profesorado, metodologías de enseñanza, razones que justifiquen el estudio de los polímeros con detenimiento y la opinión sobre los polímeros en los libros de texto.

Tabla 1. Preguntas que se formularon a los profesores sobre los polímeros y su enseñanza. P-1.-Dedicación a los polímeros: ¿Qué asignaturas impartes 0 has impartido que contengan el tema de polímeros? ¿Cuántas horas de enseñanza dedicas al año al tema de los polímeros en cada una de estas asignaturas? ¿Por qué dedicas ese número de horas?

P-2.-Opinión sobre la necesidad de ser los polímeros abordados con mayor extensión y profundidad: ¿Crees que dada la importancia de los polímeros en nuestra sociedad deberían ser abordados en el currículo de la ESO y el Bachillerato con mayor extensión y profundidad?

P-3.-Necesidad de mayores conocimientos sobre polímeros del profesorado: ¿Crees que el profesorado necesita una mayor formación en polímeros?

P-4.-Metodologías de enseñanza de los polímeros en el aula: ¿Cómo piensas que sería mejor introducir los polímeros en el aula?

P-5.-Razones que justifiquen la necesidad de estudiar polímeros: ¿Serías capaz de dar alguna razón concreta que pueda justificar la necesidad de tratar con detenimiento los polímeros en el aula?.

P-6.-Opinión sobre los polímeros en los libros de texto: ¿Qué opinas del tratamiento que hacen los libros de texto del tema de polímeros?

Las 21 preguntas (ítems) del cuestionario para el análisis de libros de texto de la Tabla 2 fueron seleccionadas y revisadas (otorgándoles, por tanto, validez de contenido) por dos profesores universitarios y dos profesores de educación secundaria tomando en consideración los conocimientos mínimos que debería tener todo ciudadano culto al terminar la Educación Secundaria Obligatoria (que están recogidos, además, en la legislación oficial).
Tabla 2. Cuestionario de análisis de libros de texto.

i-1.-¿Se menciona la revolución de los polímeros sintéticos en el campo de los materiales desde el fin de la segunda guerra mundial hasta nuestro días y cómo han ido sustituyendo a otros materiales como la madera o los metales?

Í-2.-¿Se define de forma clara los conceptos de monómero y polímero?

í-3.-¿Se realiza alguna clasificación de los diferentes tipos de polímeros con ejemplos de cada tipo?

Í-4.-¿Se relacionan los polímeros con todos los objetos de uso cotidiano presentes actualmente en la sociedad mediante dibujos o fotografías ?

í-5.-¿Se comenta brevemente el proceso de vulcanizado del caucho?

í-6.-¿Se menciona a Leo Bakeland como el primer científico que sintetizó el primer polímero sintético, la baquelita?

í-7.-¿Se explica lo que es una reacción de polimerización?

i-8.-¿Se indica como formular una reacción de polimerización y los polímeros más comunes?

I-9.-¿Se indican las propiedades únicas que poseen los plásticos que hace que se utilicen para multitud de aplicaciones?

I-10.-¿Se discute la importancia social y económica de los plásticos?

Í-11.-¿Se explican los polímeros naturales dando algunos ejemplos?

Í-12.-¿Se comenta el refuerzo con fibra en los materiales compuestos o composites?.

Í-13.-¿Se explica el procesado de los materiales plásticos: moldeado por inyección, extrusión, soplado, espumado, etc.?

Í-14.- ¿Se describe en el texto la importancia de las tres erres (3R) de ecología (reducir, reutilizar y reciclar) en el caso de los plásticos?

í-15.-¿Se explica el significado de los símbolos que acompañan a los envases de plástico, constituidos de pequeños triángulos formados por tres flechas y acompañados por un número en su interior y con letras en su parte inferior externa?

Í-16.-¿Se comenta lo que es un plástico biodegradable y sus ventajas respecto a los plásticos convencionales?

İ-17.-¿Se hace mención al premio Nobel de Química por el descubrimiento y desarrollo de los polímeros conductores en el año 2000?

í-18.-¿Se plantean cuestiones y ejercicios sobre polímeros?

Í-19.-¿Se proponen prácticas de laboratorio 
sobre polímeros?

í-20.-¿Se hace referencia en el libro de texto a páginas web con contenidos, vídeos 0 applets sobre polímeros?

Í-21.-¿Viene el libro de texto acompañado de un $C D$ con imágenes, vídeos, páginas web de referencia o animaciones sobre polímeros?

\subsection{Procedimiento}

La realización de las entrevistas a los profesores se ha llevado a cabo siguiendo el guión de preguntas (aunque no se hacen necesariamente en el orden que están escritas) expuestas en la Tabla 1.

Las entrevistas fueron registradas con una grabadora. Durante la entrevista se permitió al entrevistado la realización de matices en sus respuestas que pudieran proporcionar un valor añadido a la información que dan. A continuación, fueron transcritas en un documento y analizadas por los autores de este trabajo.

En referencia al análisis de los libros de texto, se aplicó el cuestionario de la Tabla 2 a cada uno de los libros de texto del Anexo, registrándose en una hoja (planilla) si la respuesta a la pregunta del cuestionario era "Sí", "Parcialmente", o "No". Es obvio que este análisis no pretende ser una evaluación de la calidad instruccional los libros analizados, sino que únicamente examina la presencia de conceptos y procedimientos asociados a los polímeros. La respuesta de cada ítem puede ser "Sí", "Parcialmente", o "No". Finalmente, se computan y registran "Síes", "Parcialmentes" y "Noes".

\section{RESULTADOS Y DISCUSIÓN}

\subsection{Entrevistas}

\subsubsection{Dedicación a los polímeros $(P-1)$}

De las respuestas dadas por los profesores a la primera pregunta $(\mathrm{P}-1)$ de la Tabla 1, se desprende que la dedicación a los polímeros por parte del profesorado es prácticamente nula, tanto en la ESO como en Bachillerato. Únicamente en la asignatura de Tecnología de $3^{\circ}$ de la ESO se abordan algunas cuestiones sobre polímeros (entre 2 y 4 clases, esto es, entre 2 y 4 horas como máximo).

El motivo manifestado por las profesoras del Departamento de Física y Química para no dedicar tiempo a los polímeros es que los polímeros constituyen un tema complejo que requiere tener una base química que los alumnos no llegan a alcanzar.

Una profesora de Física y Química afirmaba: "No llego a los polímeros porque no tenemos tiempo, no hay forma (...) Además, en la parte de polímeros se necesita que sepan Química Orgánica y formulación".

Por otro lado, los profesores de Física y Química indican que prácticamente nunca aparece en las pruebas de acceso a la universidad, lo que hace que lo consideren como un tema secundario.

Los dos profesores del Departamento de Tecnología están de acuerdo en que el número de horas que dedican a polímeros en la ESO es muy escaso porque el currículum es muy amplio y tampoco tienen suficiente tiempo para introducir este tema.

\subsubsection{El porqué de la introducción de los polímeros con mayor extensión $y$ profundidad (P-2 y P-5)}

Todos los profesores del Departamento de Física y Química coinciden en que sí que es necesario abordar los polímeros en mayor extensión y profundidad debido a su gran presencia en la sociedad actual, y a la necesidad de concienciar los alumnos desde el punto de vista medioambiental. En concreto, una profesora expone: "los polímeros permiten una conexión entre lo que los estudiantes se van encontrando cada día y lo que tú presentas en el aula, es decir, representan un ejemplo muy claro de las relaciones que hay entre la Ciencia, la Tecnología y la Sociedad".

Sin embargo, los profesores del Departamento de Tecnología sostienen que, aunque son muy importantes em la sociedad actual $y$, por tanto, se han enseñar, en la asignatura de Tecnología no es necesario ahondar más de lo que se hace en estos momentos porque, según un profesor: "lo que interesa es la parte técnica de los plásticos, cómo trabajarlos, diferenciarlos, reciclarlos, fabricarlos y hacer piezas con ellos pero no sus estructuras moleculares o su reactividad química"

\subsubsection{Formación en polímeros del profesorado $(P-3)$}


Todos los profesores admiten que sus conocimientos sobre polímeros son bastante limitados. No obstante, aducen que no se sienten motivados para conseguir mejorar esa formación por la poca importancia que tienen actualmente en el contexto académico actual de la educación secundaria.

\subsubsection{Enseñanza en el aula de los polímeros $(P-4)$}

Es un sentir mayoritario entre el profesorado la necesidad de emplear metodologías activas de carácter indagativo en el aula. Los profesores de Física y Química hacen hincapié en: a) Conocer los materiales de uso cotidiano que son polímeros y las implicaciones medioambientales de su uso; y b) Saber cómo se llevan a cabo las reacciones de polimerización y que los estudiantes pongan en práctica algún caso.

Por su parte, los profesores de Tecnología inciden en: a) Investigar las aplicaciones de los polímeros; y b) Identificar distintos tipos de plásticos mediante métodos físicos y químicos.

\subsubsection{Los polímeros en los libros de texto ( $P$ - 6)}

Casi todos los profesores del Departamento de Física y Química desconocen, en general, cómo se presenta el contenido de los materiales poliméricos en los libros de texto. Lo justifican por la amplitud del currículum que supone tener que dejar de lado determinados temas. Solamente la profesora que imparte la asignatura "Ciencias para el mundo contemporáneo", obligatoria para todos los estudiantes que cursan el Bachillerato, opina con conocimiento de causa que: "Son bastante flojos todos. Son libros que quieren decir algo pero se quedan a medias. Para los estudiantes de ciencias sociales y humanidades los veo bien, pero para los de ciencias son inadecuados".

Los dos profesores del Departamento de Tecnología creen que la presentación que se hace en los libros de texto de Tecnología sobre los plásticos es inapropiado.

\subsection{Análisis de libros de texto}

La aplicación del cuestionario del análisis de libros de texto (Tabla 2) a todos los textos de ESO y Bachillerato conjuntamente (Anexo) arroja unos porcentajes que pueden verse en la Figura 1.
En esta Figura 1, se observa que los libros de la ESO y Bachillerato en conjunto contienen, total o parcialmente, información sobre todos los ítems considerados fundamentales, aunque en algunos casos (8 casos) los porcentajes de "Síes" son bajos, como en los ítems 1 (revolución de los polímeros como substitutos de otros materiales), 6 (Bakeland obtiene el primer polímero sintético), 9 (propiedades específicas de los plásticos), 10 (importancia social y económica de los plásticos), 13 (procesado de los materiales plásticos: inyección, extrusión, etc.), 17 (premio Nobel por el descubrimiento y desarrollo de los polímeros conductores ), 20 (páginas web con contenidos, vídeos o applets sobre polímeros) y 21 (CD con imágenes, vídeos 0 animaciones sobre polímeros).

Figura 1. Porcentajes de respuestas (Sí, Parcialmente o No) de cada uno de los 21 ítems del análisis del libro de texto de las dos asignaturas de ESO y las tres de Bachillerato conjuntamente.

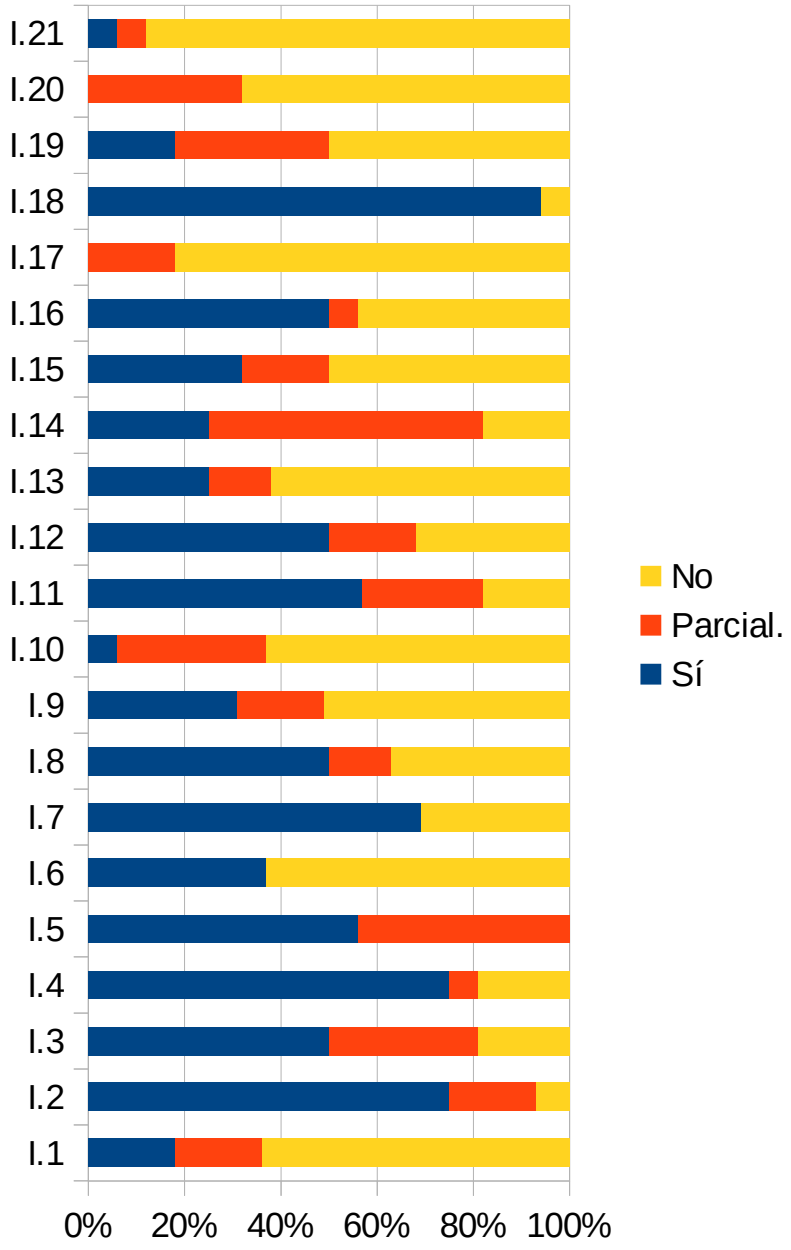


En la Tabla 3 se presentan los porcentajes medios de respuestas (Sí, Parcialmente y No) en el análisis de los libros de texto de cada una de las asignaturas por separado y en conjunto. De esta Tabla 5 se destacan varias cosas:

a) Que la asignatura optativa Tecnología Industrial de primero de Bachillerato tiene el porcentaje más alto de "Síes", y es la única asignatura que contiene más del 50\% (57,14\%) de respuestas "Sí" a los 21 ítems de la plantilla de análisis.

b) Que dados los porcentajes de contenidos que no recogen los libros de texto de la ESO $(44,44 \%)$, y teniendo presente que en la ESO acaba la educación obligatoria (muchos estudiantes se van al mercado laboral) no hay garantías de que se puedan formar ciudadanos alfabetizados científicamente en este aspecto.

c) Que incluso los estudiantes que acaban el Bachillerato científico tampoco tienen la posibilidad de lograr formarse de manera adecuada en estos materiales básicos en nuestra sociedad $(43,45 \%$ de contenidos que no están presentes a lo largo de toda su educación secundaria).

d) Que la asignatura "Ciencias para el Mundo Contemporáneo", que pensamos debería asumir la formación en estos contenidos en el Bachillerato (tanto para los que estudian ciencias, como para los que estudian humanidades o ciencias sociales), tampoco está cumplimiendo realmente su función $(53,57 \%$ de "Noes" en el cuestionario pone en evidencia que no se abordan convenientemente estos conceptos).

Tabla 3. Porcentajes medios de respuestas en el análisis de los libros de texto de cada asignatura por separado y en conjunto.

\begin{tabular}{|c|c|c|c|}
\hline Asignatura & sí & P. & No \\
\hline Tecnologías $3^{\circ} \mathrm{ESO}$ & 33,33 & 19,05 & 47,62 \\
\hline Física y Química $4^{\circ} E S O$ & 38,09 & 19,05 & 42,86 \\
\hline Ciencias M. C. $1^{\circ} \mathrm{BAC}$ & 27,38 & 19,05 & 53,57 \\
\hline Tecnología Ind. $1^{\circ} \mathrm{BAC}$ & 57,14 & 14,29 & 28,57 \\
\hline Química $2^{\circ} \mathrm{BAC}$ & 46,43 & 14,29 & 39,28 \\
\hline Todas de la ESO & 36,51 & 19,05 & 44,44 \\
\hline Todas de la ESO y BAC & 39,29 & 17,26 & 43,45 \\
\hline
\end{tabular}

Por otro lado, son dignos de ser subrayados los siguientes datos:

a) Hemos detectado que en muchos de los libros de texto (más del 60\%), tanto de ESO como de Bachillerato, se habla de polímeros dando a entender que se trata de un sinónimo de plástico, cuando los plásticos son únicamente uno de los tipos de polímeros que existen. En este sentido, es muy importante señalar que en sólo en el $50 \%$ de los libros de texto se explica claramente que existen diferentes tipos de polímeros (Ítem 3 de la Figura 1), lo cual podría contribuir a inducir concepciones erróneas entre el alumnado.

b) Más del $60 \%$ de los libros de texto no menciona nada del procesado de los materiales plásticos, esto es, del moldeado por inyección, extrusión, soplado, etc. (Ítem 13 de la Figura 1).

c) Alrededor del $75 \%$ de los textos no incluye información alguna sobre la importancia de los polímeros conductores, que se vio reflejada en forma de Premio Nobel para los tres descubridores (Alan J. Heeger -USA-, Alan G. MacDiarmid -EEUU y Nueva Zelanda- y Hideki Shirakawa -Japón-).

Estos últimos datos podrían justificar, al menos en parte, los resultados obtenidos por Serrano-Aroca y Solaz-Portolés (2014) en entrevistas a estudiantes de educación secundaria (ESO y Bachillerato), que muestran que:

a) Una buena parte de los estudiantes entrevistados tiene dificultades para definir polímero y sus tipos, y creen que todos los polímeros son plásticos.

b) Prácticamente ninguno sabe en qué consiste la inyección y la extrusión de plásticos.

c) Ninguno puede aportar información alguna sobre polímeros conductores, su importancia o sus descubridores

\section{CONCLUSIONES}

De los resultados obtenidos en las entrevistas al profesorado participante en este estudio pueden extraerse varias conclusiones en relación con los polímeros y su enseñanza (siempre teniendo presentes las limitaciones de nuestra investigación: pocos sujetos y del mismo centro educativo que imposibilitan efectuar generalizaciones con márgenes de fiabilidad 
elevados):

a) Se admite que su presencia en el currículo es casi nula.

b) Se asume su relevancia social e interés $y$, consecuentemente, la necesidad de estar presente en el currículo.

c) Se reconoce la falta de formación del profesorado.

d) Se cree que una metodología de carácter indagativo con trabajos prácticos sería la mejor manera de trabajar en el aula.

d) Se denuncia la poca calidad instruccional de los libros de texto.

Respecto al tratamiento ofrecido por los libros de texto de la educación secundaria, el análisis de textos efectuado en este estudio no resulta muy alentador. Así, 8 de los 21 aspectos analizados en los textos que, según expertos, son esenciales, no son abordados en más del $50 \%$ de los textos. Además, el $43 \%$ de dichos contenidos básicos no se recoge en los libros analizados. Todo ello apunta en la dirección de que este recurso educativo tampoco está en condiciones de facilitar los conocimientos adecuados sobre polímeros.

De la información obtenida de profesores y libros de texto parece que estamos en condiciones de dar respuesta, siquiera sea en primera aproximación, a la pregunta de investigación que ha guiado este trabajo. La respuesta es que sí, se puede decir que, en gran medida, el bajo conocimiento sobre polímeros de los estudiantes españoles de educación secundaria está justificado por el proceder del profesorado y el tratamiento que se da en los libros de texto de este tema.

No obstante, se debe recalcar como aspecto positivo de este estudio la loable actitud del profesorado hacia un cambio en la enseñanza de los polímeros. Los profesores son conscientes de que no están proporcionando a los estudiantes la formación que posibilita una mejor comprensión del mundo que les rodea, a ser consumidores críticos y responsables, o ser activistas en favor del medio ambiente. Esto es, saben que estan limitando la alfabetización científica de la ciudadanía (De Oliveira et al., 2013). Además, apuestan por metodologías de enseñanza de carácter indagativo en el aula, que son las que, según las investigaciones en la didáctica de las ciencias, tienen mejores resultados (Acar \& Tarhan, 2006; Furió \& Furió, 2009)

\section{AGRADECIMENTOS}

Agradecemos a los profesores del Instituto de Educación Secundaria Isabel de Villena de Valencia su amable participación en este estudio y a la dirección del Instituto por permitir el análisis de sus libros de texto.

\section{REFERENCIAS}

1. De Pro, A. Alambique. Didáctica de las Ciencias Experimentales, 2007, 53, 3.

2. Caamaño, A. Alambique. Didáctica de las Ciencias Experimentales, 2009, 59, 9.

3. Caamaño, A. Educación Química, 2006, $17,2$.

4. Serrano-Aroca, A., Solaz-Portolés, J.J. Periódico Tche Química, 2014, 11, 47. Disponível www.periodico.tchequimica.com, accesado en 7/01/2015.

5. Enkvist, I., Bordón, 2010, 62, 49.

6. Yore, L. D., Journal of Research in Science Teaching, 1991, 28, 55.

7. Duarte, M. C., Revista Portuguesa de Educação, 1999,12, 227.

8. Campanario, J. M., Enseñanza de las Ciencias, 2001, 19, 351.

9. Perales, F. J., Jiménez, J. D., Enseñanza de las Ciencias, 2002, 20, 369.

10. De Pro, A., Sánchez, G., Valcárcel, M. V. Enseñanza de las ciencias, 2008, 26 , 193.

11. Moreira, M. A., Lang de Silveira, F. Instrumento de pesquisa em ensino e aprendizagem, EDIPURCS, 1993, Porto Alegre.

12. Mayan, M.J. (2001) An introduction to qualitative methods: A training module for students and professionals, Qual Institute Press, 2001, Edmonton, Canada.

13. González, M. T., Sierra, M. Enseñanza de las ciencias, 2004, 22, 389.

14. Serrano-Aroca, A., Solaz-Portolés, J.J. op. Cit.

15. De Oliveira, D., Delourdes, M., Pereira, R., Vázquez-Alonso, A. Revista Electrónica de Enseñanza de las

PERIÓDICO TCHÊ QUÍMICA • www.periodico.tchequimica.com • Vol. 12 N. 24 - ISSN 1806-0374 (impresso) • ISSN 1806-9827 (CD-ROM) • ISSN 2179-0302 (meio eletrônico) 
Ciencias, 2013, 12, 313.

16. Acar, B., Tarhan, L. International Journal of Science and Mathematics Education, 2006, 5, 343.

17. Furió, C., Furió, C. Educación Química, 2009, 20, 246.

\section{ANEXO}

7.1. Listado de libros de texto de E.S.O analizados en las distintas asignaturas

\subsubsection{Asignatura: Tecnologías $3^{\circ}$ E.S.O}

[1] Romero Quílez, A. y Serrate i Cunill, X. (2011). Tecnologías serie conocer. Educación Secundaria Obligatoria 3. Editorial Bruño: Madrid. [2] Armada Simancas, M., Blanco Gil, R., Cabezas Gómez, E., Blé Berrio, M., López de Guereñu, J.G., García Monge, J.A., López Soriano, T., López Werner, J., Ortiz Gandía, M.I., Peña Pérez, A., Pérez Malagón, J., Vallejo Martín-Albo, C. y Villanueva García, O.(2007). Tecnologías 3 ESO. Proyecto La Casa del Saber. Santillana Educación, S.L.: Madrid.

\subsubsection{Asignatura: Física y Química $4^{\circ}$ E.S.O}

[1] García Pozo, T. y Equipo Edebé. (1996). Área de Ciencias de la Naturaleza (Física y Química). Segundo ciclo de Educación Secundaria Obligatoria. Edebé: Barcelona.

[2] Caamaño, A., Obach, D. y Pérez Redón, E. (2005). Física i Química. Ciències de la Naturalesa. Segon Cicle 4. Pojecte @ula. Educació Secundària Obligatòria. Editorial Teide: Barcelona.

[3] Vidal Fernández, M.C., De Prada P. de Azpeitia, F. y Sanz Martínez, P. (2008). Física i Química 4 ESO. Projecte La Casa del Saber. Edicions Voramar, S.L./ Santillana Educación, S.L.: València.

[4] Cañas, A., Puente, J., Remacha, M. y Viguera, J.A. (2008). Física i Química. ESO 4. Projecte Secundària. Ediciones SM: Navarra.

7.2. Listado de libros de texto de Bachillerato analizados en las distintas asignaturas

\subsubsection{Asignatura: Tecnología Industrial $1^{\circ}$ Bachillerato}

[1] Fidalgo Sánchez, J.A., Fernández Pérez, M.R., Fernández Fernández, N. y Gutiérrez Álvarez, E.R. (2002). Tecnología Industrial bachillerato. Editorial Everest, S.L.: León.

[2] Silva Rodríguez, F.(2008). Tecnología Industrial Bachillerato $1 . \quad$ McGraw Hill/Interamericana de España, S.A.U.: Madrid.

\subsubsection{Asignatura: Ciencias para el Mundo Contemporáneo $1^{\circ}$ Bachillerato}

[1] Rubio, N., Pulido, C. y Roiz, J.M. (2008). Ciencias para el Mundo Contemporáneo. bachillerato 1.Grupo Anaya, S.A. : Madrid.

[2] Fornells, M., Gómez, S., Jimeno, A., Liras, S., Pérez, E. y Rodríguez, A. (2008). Ciències per al món contemporani batxillerat. Editorial Casals, $\mathrm{S}$. A.: Barcelona.

[3] Anguita Virella, F., Carrión Vázquez, M., Cerezo Gallego, J.M., Henche Ruiz, A.I., Hidalgo Moreno, A.J., López de Guereñu, J.G. y Peña Pérez, A. (2008). Ciencias para el mundo contemporáneo 1 bachillerato. Proyecto la Casa del Saber. Santillana Educación, S.L.: Madrid.

[4] Pedrinaci, E., Gil C., De Dios Jiménez, J., Puente, J. y Pedreira, S. (2009). Ciencias para el mundo contemporáneo 1 bachillerato. Ediciones SM: Madrid.

\subsubsection{Asignatura: Química $2^{\circ}$ Bachillerato}

[1] Quílez, J. Lorente, S., Sendra, F. y Enciso, E. (2009). Afinidad Química bachillerato 2. Editorial ECIR,S.A: Valencia.

[2] Ballester, J.R., Ballester, M.E., Calatayud, M.L., Sabater, J.V. y Trilles, E.(2011). Química Segon de Batxillerat Colecció Quarkonio. NAU llibres: València.

[3] Guardia Villaroel, C., Menéndez Hurtado, A.I. y De Prada P. De Azpeitia, F. (2009). Química 2 Batxillerat. Projecte La Casa del Saber. Edicions Voramar, S.L.I Santillana Educación, S.L.: València.

[4] Del Barrio, J.I., Bárcena, A.I., Sánchez, A. y Caamaño, A. (2009). Química 2 Batxillerat. Ediciones SM: Madrid. 